Harald Lüngen/Marc Kupietz (Mannheim)

\title{
IBK- und Social Media-Korpora am Leibniz-Institut für Deutsche Sprache
}

\begin{abstract}
Der Beitrag untersucht vorhandene Lösungen und neue Möglichkeiten des Korpusausbaus aus Social Media- und internetbasierter Kommunikation (IBK) für das Deutsche Referenzkorpus (DEREKo). DEREKo ist eine Sammlung gegenwartssprachlicher Schriftkorpora am IDS, die der sprachwissenschaftlichen Öffentlichkeit über die Korpusschnittstellen COSMAS II und KorAP angeboten wird. Anhand von Definitionen und Beispielen gehen wir zunächst auf die Extensionen und Überlappungen der Konzepte Social Media, Internetbasierte Kommunikation und Computer-mediated Communication ein. Wir betrachten die rechtlichen Voraussetzungen für einen Korpusausbau aus Sozialen Medien, die sich aus dem kürzlich in relevanten Punkten reformierten deutschen Urheberrecht, aus Persönlichkeitsrechten wie der europäischen Datenschutz-Grundverordnung ergeben und stellen Konsequenzen sowie mögliche und tatsächliche Umsetzungen dar. Der Aufbau von Social Media-Korpora in großen Textmengen unterliegt außerdem korpustechnologischen Herausforderungen, die für traditionelle Schriftkorpora als gelöst galten oder gar nicht erst bestanden. Wir berichten, wie Fragen der Datenaufbereitung, des Korpus-Encoding, der Anonymisierung oder der linguistischen Annotation von Social Media-Korpora für DEREKo angegangen wurden und welche Herausforderungen noch bestehen. Wir betrachten die Korpuslandschaft verfügbarer deutschsprachiger IBK- und Social Media-Korpora und geben einen Überblick über den Bestand an IBK- und Social Media-Korpora und ihre Charakteristika (Chat-, Wiki Talk- und Forenkorpora) in DEREKo sowie von laufenden Projekten in diesem Bereich. Anhand korpuslinguistischer Mikro- und Makro-Analysen von Wikipedia-Diskussionen im Vergleich mit dem Gesamtbestand von DEREKo zeigen wir charakterisierende sprachliche Eigenschaften von Wikipedia-Diskussionen auf und bewerten ihren Status als Repräsentant von IBK-Korpora.
\end{abstract}

\section{Einleitung: Definitionen}

Die drei Konzepte Social Media, Internetbasierte Kommunikation und Computermediated Communication werden in der Forschung auf den ersten Blick nebeneinander scheinbar für ein und dasselbe Kommunikationsgenre verwendet. Auch auf der Jahrestagung des Leibniz-Instituts für Deutsche Sprache (IDS) 2019 werden 
alle drei Begriffe gebraucht, wobei einzelne Forscher oder Forscherinnen in der Regel einen von ihnen primär verwenden. Inwieweit stimmen die drei Konzepte genau überein, und worin unterscheiden sie sich möglicherweise? Im Social Media Guide for Researchers definieren Cann/Dimitriou/Hooley (2011) Social Media als „Internet services where the online content is generated by the users of the service“. Im Erläuterungstext werden zwei weitere Merkmale angeführt, die wir auch als definitorisch verstehen: Erstens handelt es sich bei Social Media um Dienste des sogenannten Web 2.0, welches seit dem ersten Jahrzehnt dieses Jahrhunderts existiert - ältere Formen wie Internet Relay Chat (IRC), Usenet News oder E-Mail sind somit ausgeschlossen. Und zweitens handelt es sich um Dienste, die der Kommunikation, der Kollaboration oder dem Teilen und Rezipieren von multimedialen Inhalten dienen. ${ }^{1}$

Als internetbasierte Kommunikation (IBK) bezeichnet Angelika Storrer (2018) den „Forschungsgegenstand, der sich mit Technologien, Prozessen und Produkten der Kommunikation unter Nutzung der technischen Infrastruktur des Internets beschäftigt“. Somit ist der Gegenstand von IBK weiter gefasst als der von Social Media, da IBK auf das Internet, d.h. auf diejenigen Technologien, die auf dem Protokoll TCP-IP beruhen, verweist, welche neben dem World Wide Web (WWW) auch E-Mail und das Usenet umfassen. Auf der anderen Seite zählen Technologien wie SMS, die auf dem Telefonnetz beruhen, nicht zu IBK, wie auch nicht zu Social Media. Ebenso stellen wir fest, dass in Storrers Definition nur die Kategorie Kommunikation angeführt wird, während in der Definition von Social Media wie oben wiedergegeben außerdem Kollaboration und Multimedia-Inhalte eine Rolle spielen.

Der älteste der drei Begriffe ist Computer-mediated Communication, er wurde von Susan Herring geprägt. Ihre Definition in Herring (2007) lautet: CMC ist ,predominantly text-based human-human interaction mediated by networked computer or mobile telephony“. Zwei Aspekte erscheinen hier wichtig, erstens verweist „networked Computer“ nicht zwingend nur auf das Internet als Netzwerk, und zweitens wird Telefonie explizit eingeschlossen. ${ }^{2}$

1 Wir danken den Kolleginnen und Kollegen aus der IDS-Abteilung Digitale Sprachwissenschaft, die uns mit ihrer Expertise und mit Ergebnissen für diesen Beitrag unterstützt haben: Peter Fankhauser, Peter M. Fischer, Paweł Kamocki und Eliza Margaretha-Illig.

2 In früheren Versionen der Definition wurde Telefonie nicht eigens genannt, z.B. Herring (2004, S. 27). 


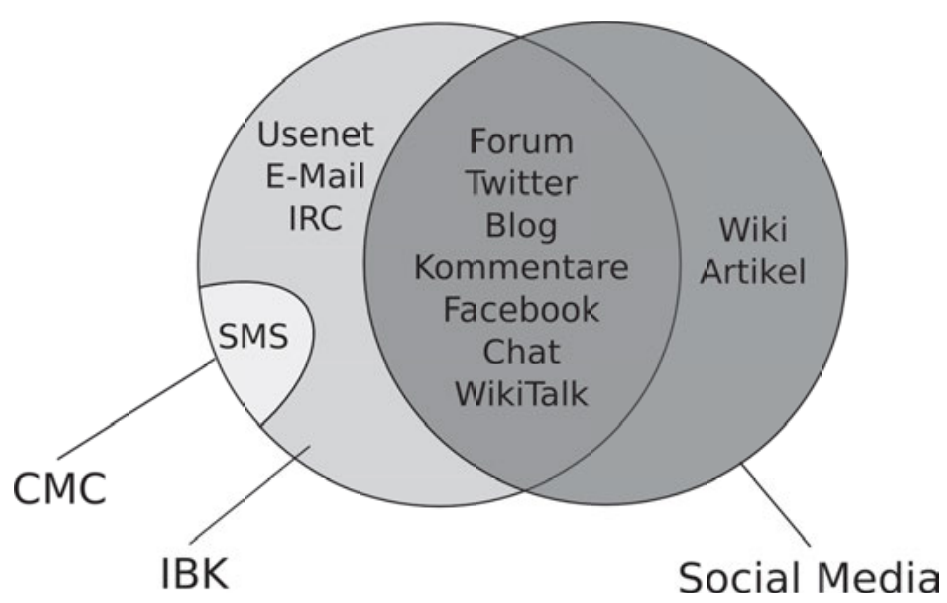

Abb. 1: Extensionen von CMC, IBK und Social Media

In Abbildung 1 haben wir diejenigen Plattformen bzw. Kommunikationsformen, von denen im weiteren Verlauf des Artikels hauptsächlich die Rede sein soll, einmal diesen drei Konzepten zugeordnet. Auf der linken Seite ist zu sehen, dass IBK im Prinzip deckungsgleich mit CMC ist, mit der Ausnahme, dass SMS-Kommunikation streng genommen nicht zu IBK gehört. Social Media ist aber erkennbar nicht synonym zu CMC, erstens weil die älteren Dienste Usenet, E-Mail und IRC auf der linken Seite nicht unter Social Media fallen, und zweitens weil Wiki als klassisches Web 2.0-Medium für das kollaborative Verfassen von Wiki-Seiten bzw. -Artikeln standardmäßig als Social Media verstanden wird, als IBK/CMC aber nur die Kommunikationskomponente Wiki Talk (auf Deutsch meist: Wiki-Diskussionen) - daher die Außenstellung von Wiki-Artikeln in Abbildung 1.

Soweit unser Verständnis dieser drei Termini insbesondere im Hinblick auf Korpora. In der Praxis verwenden wir IBK auch als deutsche Übersetzung von CMC.

\section{Voraussetzungen für IBK-Korpora in DEREKo}

In diesem Abschnitt untersuchen wir die Voraussetzungen und Möglichkeiten von Korpora Internetbasierter Kommunikation am IDS, genauer gesagt, für die Korpora der deutschen Schriftsprache der Gegenwart, die unter dem Namen Deutsches Referenzkorpus (DeReKo) durch den Programmbereich Korpuslinguistik angeboten werden. Im Rahmen der Schriftkorpora sind solche Korpora Internetbasierter Kommunikation und Sozialer Medien interessant, die nicht auf 
(Transkripten von) Audio- oder Video-Inhalten beruhen - derartige Korpora werden in der IDS-Abteilung Pragmatik aufgebaut und genutzt (vgl. Marx/Schmidt/ Neise in diesem Band).

DEReKo ist die größte linguistisch motivierte Sammlung deutschsprachiger Texte mit über 42 Milliarden Tokens (Stand Juli 2019) aus Presse, Belletristik, wissenschaftlichen und populärwissenschaftlichen Artikeln, Gebrauchstexten, Plenardebattenprotokollen und vielen weiteren Genres. Sie wird seit 1964 kontinuierlich aufgebaut und enthält Texte der Gegenwartssprache, die 1956 oder später verfasst wurden. DEREKo ist mehrfach morphosyntaktisch und syntaktisch annotiert (z.B. POS-Tagging, Konstituenz- und Dependenzanalysen). DeReKo ist rechtlich abgesichert durch mehr als 120 Lizenzverträge mit Rechteinhabern, 98\% der Daten sind über die Korpusrechercheschnittstellen für wissenschaftliche Zwecke weltweit öffentlich nutzbar. DEREKo stellt somit eine Stichprobe des gegenwärtigen Schriftsprachgebrauchs dar und dient der germanistischen Sprachwissenschaft als empirische Grundlage überall dort, wo die Sprache der Gegenwart und jüngeren Vergangenheit Gegenstand der Forschung ist, vor allem in quantitativen Untersuchungen, die große Korpora benötigen (Kupietz et al. 2018). Für die meisten Nutzer erfolgt der Zugriff auf DEReKo über die beiden Korpusrechercheschnittstellen des IDS, COSMAS II (Abb. 2; seit 1994, vgl. Bodmer Mory 2014) und KorAP (Abb. 3; seit 2016, vgl. Diewald et al. 2016).

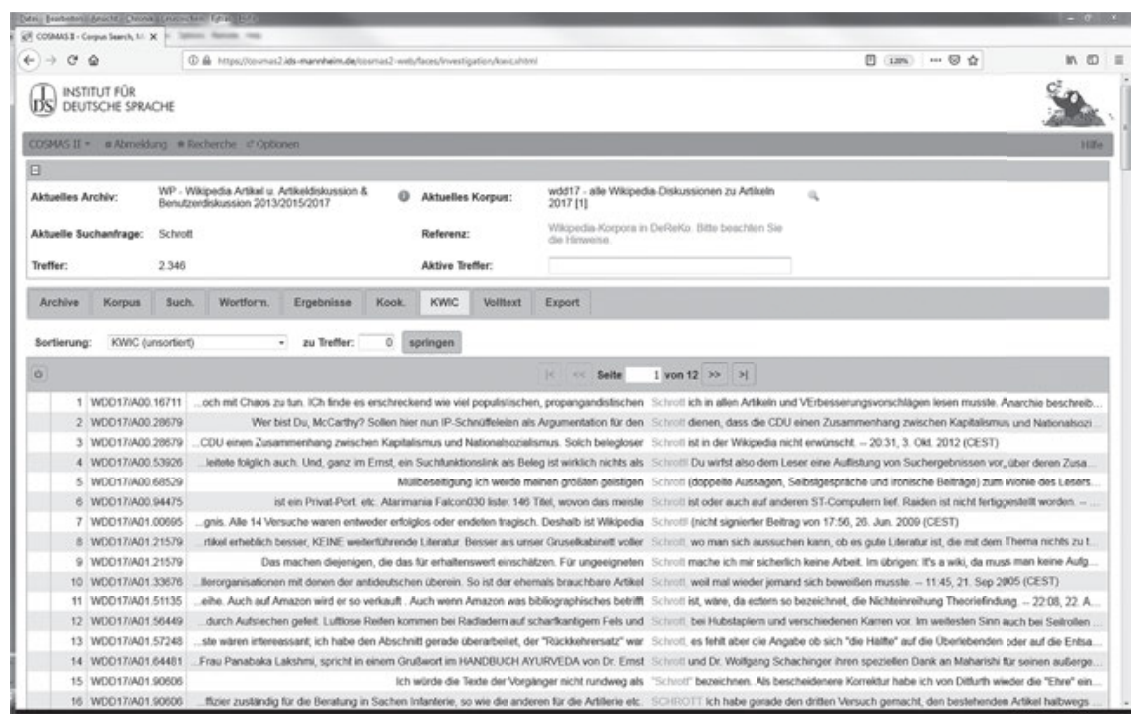

Abb. 2: Korpusrechercheschnittstelle COSMAS II 


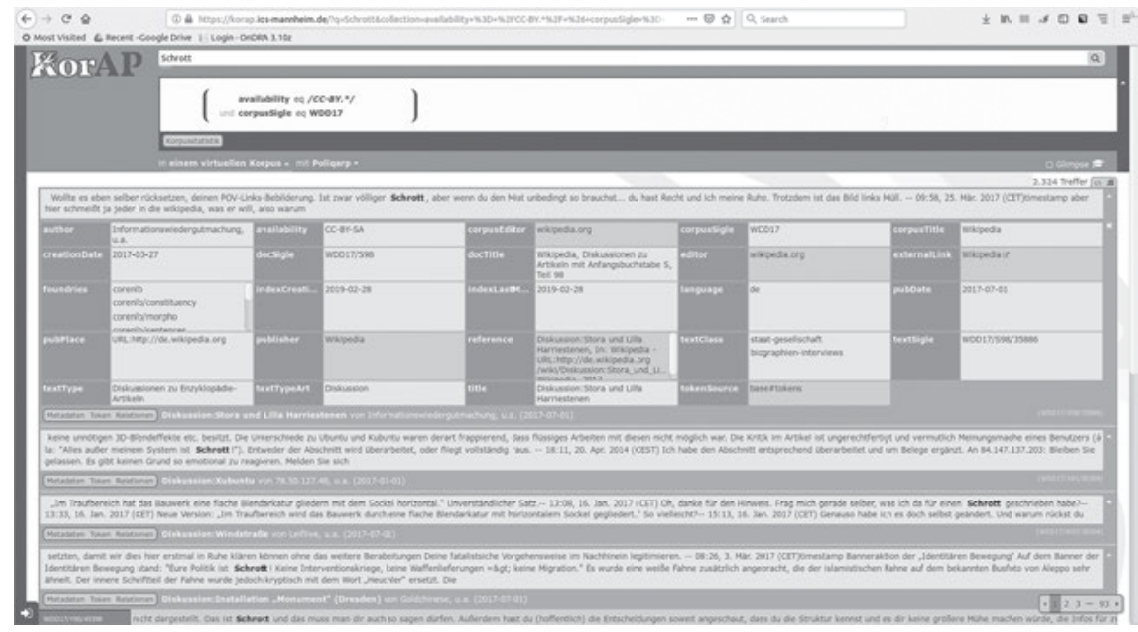

Abb. 3: Korpusrechercheschnittstelle KorAP

DEREKo möchte Daten Internetbasierter Kommunikation sampeln, die rechtlich einwandfrei als Korpus einem erweiterten, wissenschaftlichen Nutzerkreis zur Verfügung gestellt werden können. Der Aufbau derartiger Korpora tangiert Urheberrechte und allgemeine Persönlichkeitsrechte in besonderer Weise und anders als traditionelle Pressekorpora (Kamocki/Kinder-Kurlanda/Kupietz 2016). Im Folgenden stellen wir die rechtlichen Voraussetzungen für IBK-Korpora dar und untersuchen die wichtigsten deutschsprachigen frei verfügbaren IBK-Korpora sowie einige einschlägige IBK-Korpora anderer europäischer Sprachen daraufhin, wie mit diesen Voraussetzungen strategisch und technisch umgegangen wird.

\subsection{Urheberrechtliche Voraussetzungen}

Auch IBK-Nutzerbeiträge (Posts) sind potenziell durch das Urheberrecht geschützt. Voraussetzung dafür ist, dass ein Post eine Schöpfungshöhe erreicht, also für sich ein „Werk” darstellt (§ 2 UrhG). Ob ein Text eine Schöpfungshöhe aufweist, hängt nicht unbedingt von seiner Länge ab, dies kann letztlich nur aufgrund seines konkreten Inhalts beurteilt werden. Für bestimmte Genres kann man aber Tendenzen aufzeigen. In dem Rechtsgutachten, das im Projekt zur Kuratierung des Dortmunder Chatkorpus für CLARIN-D-Infrastrukturen erstellt wurde, kamen die Anwälte zu dem Schluss, dass Chat-Posts in der Regel keine Schöpfungshöhe aufweisen, da sie nicht individuell genug sind (Beißwenger et al. 2017a). Twitter- 
Kommunikation scheint aber stärker zur Schöpfungshöhe zu neigen, schon allein dadurch dass Twitter grundsätzlich öffentlich ist. Twitter-Kommunikation ist insgesamt monologischer und weniger interaktiv ausgerichtet als Chat - einige User twittern Gedichte, einige positionieren sich sogar explizit als Autoren. ${ }^{3}$ Es fällt auch auf, dass Twitter-Posts nicht standardmäßig zu einer abgegrenzten Interaktion gehören, die auch durch ein eigenständiges Dokument analog einem ChatLogfile oder einer Webseite mit einem Blog-Beitrag und seinen Kommentaren repräsentiert wird. All dies spricht dafür, dass Tweets ein weitaus höheres Potenzial für eine gewisse Schöpfungshöhe und sogar für einen Status als eigenständige Texte aufweisen.

Was sind nun die Strategien von IBK-Korpusprojekten für einen rechtlich sicheren Umgang mit dem Urheberrecht? Eine Möglichkeit ist eine gezielte Akquisition von Daten, die bereits unter einer liberalen Lizenz publiziert wurden womit die Autoren dann bereits ihre Erlaubnis erteilt haben, dass ihre Texte gesampelt und wiederveröffentlicht werden dürfen. Eine solche Strategie wurde technisch für das Blog-Korpus der Berlin-Brandenburgischen Akademie der Wissenschaften (BBAW) umgesetzt, für das genau solche Wordpress-Blogs aus dem Web gesammelt wurden, die mit einer liberalen Creative Commons-Lizenz ausgewiesen sind (Barbaresi/Würzer 2014). Dies gilt aber auch für jegliche Wikipedia-Korpora (Margaretha/Lüngen 2014; Poudat et al. 2015; Ho-Dac/Laippala 2017; Fišer/Ljubešić/Erjavec 2018), da Texte auf dieser Plattform grundsätzlich unter der Lizenz CC-BY-SA 3.0 veröffentlicht werden.

Eine zweite Möglichkeit besteht in einer Einholung von Nutzungsrechten von jedem einzelnen beteiligten Autor. Obwohl dies der aufwändigste Weg ist, wird er von den meisten, auch größeren Projekten weiterhin eingeschlagen, da nichts anderes übrig bleibt, will man auf bestimmte Daten nicht verzichten. In der Konsequenz heißt das auch, dass bestimmte Ausschnitte einer Interaktion evtl. nicht in das Korpus aufgenommen werden können, wenn die Zustimmung eines Beteiligten am Ende nicht vorliegt. Für dieses einwandfreie Vorgehen stehen z.B. das Südtiroler Korpus DiDi („Digital Natives - Digital Immigrants“, Frey/Glaznieks/ Stemle 2016), das SciLog-Korpus an der JLU Gießen (Grumt Suárez/Karlova-Bourbonus/Lobin 2016), die Korpora WhatsApp Switzerland (Ueberwasser/Stark 2017) und WhatsApp Deutschland (Siebenhaar 2018) sowie das Chat-Korpus MoCoDa2 (Mobile Communication Database, Beißwenger et al. 2018).

Eine dritte Strategie liegt vor, wenn Korpora aufgebaut werden, für die Nutzungsrechte nur von einem zentralen Ansprechpartner erworben werden müssen. Das ist immer dann der Fall, wenn die Autorinnen und Autoren einem Social Media-

3 Wir danken Konstanze Marx, die uns auf diese Argumente aufmerksam gemacht hat. 
Service-Provider oder Plattformbetreiber umfangreiche Nutzungsrechte eingeräumt haben, was oftmals durch die Zustimmung zu AGBs (Allgemeinen Geschäftsbedingungen) bei der Registrierung zu solchen Diensten erfolgt. Dann reicht es für den Entwickler eines Korpus aus, sich an einen solchen Betreiber (beispielsweise Facebook, Inc. oder Twitter, Inc.) zu wenden, um die erforderlichen Lizenzen auszuhandeln. Hierfür fanden wir im Bereich linguistische Korpora allerdings keine Beispiele, vermutlich deswegen, weil solche Betreiber oftmals einen gewissen Geldbetrag für Nutzungsrechte verlangen.

Die vierte Möglichkeit wäre die Ausnutzung der „Text- und Data-Mining“Schranke (TDM-Schranke) in der Neufassung des deutschen Urheberrechts von 2018 (§ 60d). Auch hier ist derzeit noch kein Projekt bekannt, das sich darauf beruft. Für DEREKo ist diese Schranke ohnehin nicht anwendbar, da diese allenfalls das Anlegen von temporären Kopien ohne die Möglichkeit einer öffentlichen Verfügbarmachung erlaubt.

Als fünfte nennen wir die Möglichkeit der Einholung eines Rechtsgutachtens, in dem die Unbedenklichkeit einer Wiederveröffentlichung von Daten ggf. unter bestimmten Bedingungen bescheinigt wird. Durch die Anwaltshaftung bekommt der Korpusbetreiber eine gewisse Sicherheit. Dies ist der Weg, der in dem CLARIN-D-Kurationsprojekt zum Dortmunder Chatkorpus gegangen wurde. Darin handelte es sich um Daten aus den Nullerjahren aus Plattformen, die zum großen Teil nicht mehr existieren. Für aktuelle Korpusprojekte wird sich diese Strategie selten lohnen.

Schließlich gehen einige Korpusprojekte - sechste Möglichkeit - den Weg, nicht die Primärdaten der wissenschaftlichen Öffentlichkeit zur Verfügung zu stellen (eben weil keine Genehmigung vorliegt), dafür aber die entsprechenden Werkzeuge, die zum Download und zur Aufbereitung des Korpus verwendet wurden (Ljubešić/Erjavec/Fišer 2017; Scheffler 2017). Dies ist ein attraktives Vorgehen für Twitter-Projekte, denn die Tweet-IDs, welche einen Tweet eindeutig zu identifizieren und über die Twitter-API herunter zu laden erlauben, dürfen offiziell veröffentlicht werden. Somit können Korpusnutzer in die Lage versetzt werden, die zu einer Liste von Tweet-IDs gehörenden Tweets für den persönlichen wissenschaftlichen Gebrauch selbst herunterzuladen (so sie noch auf Twitter vorhanden sind) und das (im Idealfall identische) Korpus oder Datenset selbst herzustellen.

Unser Zwischenfazit zum Umgang mit Urheberrecht für IBK-Inhalte in DEREKo lautet: Falls festgestellt wird, dass die Posts in einem Genre, für das ein Korpus zusammengestellt werden soll, zu einem hohen Anteil eine eigene Schöpfungshöhe aufweisen (letztlich aber auch, wenn man gar nicht die Mittel hat, dies zu überprüfen), gilt, dass für eine Nutzung in einem Referenzkorpus wie DEREKo die Einwilligung aller Autoren direkt oder indirekt vorliegen muss. Wie auch bei tra- 
ditionellen Pressekorpora müssen Lizenzen eingeholt werden, auch wenn viele IBK-Inhalte anders als Verlagsarchive scheinbar frei im Web verfügbar sind.

\subsection{Datenschutzrechtliche Voraussetzungen}

Der andere Bereich rechtlicher Voraussetzungen für den Aufbau von IBK-Korpora sind Persönlichkeitsrechte, darunter insbesondere Datenschutzrechte, die in der neuen (2018) EU-Datenschutz-Grundverordnung (DSGVO) sowie schon immer im Bundesdatenschutzgesetz und den Landesdatenschutzgesetzen formuliert sind: Die Erhebung, die Nutzung und die Veröffentlichung von personenbezogenen Daten bedürfen der Zustimmung eben dieser Personen. Personenbezogene Daten sind neben Metadaten über die Teilnehmer von IBK-Interaktionen auch sensible Referenzen in den Nutzerbeiträgen, welche dazu geeignet sind, Privatindividuen, die thematisiert werden, eindeutig zu identifizieren.

Die folgende Strategien hinsichtlich des Umgangs mit Datenschutz wurden in IBK-Korpusprojekten umgesetzt: Zum einen eine automatische Anonymisierung der vorkommenden Personennamen und Firmennamen durch Named Entity Recognition (NER) wie im slowenischen IBK-Korpus JANES (Fišer/Erjavec 2018). Die Autoren weisen darauf hin, dass diese computerlinguistische Anwendung nicht fehlerfrei funktioniert, sehen aber das Risiko, dass jemand dadurch in seinem Persönlichkeitsrecht geschädigt wird, für ihr Korpus als gering an. Grundsätzlich ist dazu zu sagen, dass eine Anonymisierung von Namen eigentlich nicht ausreicht, da sensible Referenzen mehr als Namen umfassen. Andererseits erscheinen viele Namen von Personen des öffentlichen Lebens (Politiker, Sportler, „Celebrities“) in IBK-Texten, die gar nicht anonymisiert zu werden brauchen.

Rechtlich sicherer und von den meisten Projekten praktiziert (z.B. Frey/Glaznieks/Stemle 2016; Ueberwasser/Stark 2017; Beißwenger et al. 2017a; Siebenhaar 2018), obwohl am aufwändigsten, ist das manuelle oder semi-automatische Anonymisieren von sensiblen Referenzen, wie es bei mündlichen Korpora Routine ist (vgl. DFG Handreichung 2013a).

Als innovativ und vielversprechend betrachten wir die Methode, die für das Korpus elektronischer Kurznachrichten MoCoDA2 angewandt wird: Die textspendenden Nutzer legen, assistentengestützt und in einer Online-Umgebung, selbst eine Pseudonymisierungstabelle für ihre Chats an (Beißwenger et al. 2018).

Schließlich, als vierte Möglichkeit, erfolgt in bestehenden Wikipedia-Korpora gar keine Anonymisierung, da hier zum einen offenbar davon ausgegangen wird, dass die Nutzer, die ja im urheberrechtlichen Sinne einer freien Nutzung zugestimmt haben, implizit auch der Nutzung ihrer Signatur und der Daten ihres Pro- 
fils zugestimmt haben. Zum anderen könnten dadurch, dass Wikipedia ein offen zugängliches Webportal ist, in dem auch die vollständige Editierungsgeschichte der Wiki-Seiten abrufbar ist, anonymisierte Informationen jederzeit mit solch geringem Aufwand rekonstruiert werden, dass das Risiko, dass hier durch die fehlende Anonymisierung ein wirklicher Schaden entsteht, sehr gering ist. Sollte ein Betroffener verlangen, dass ihn betreffende persönliche Daten nachträglich maskiert werden sollen, wird dem nachgegeben; dieses Vorgehen ist Konsens (vgl. DFG-Handreichung 2013b).

\section{Korpustechnologische Herausforderungen}

Neben den rechtlichen Bedingungen besitzen IBK-Korpora aufgrund ihrer sprachlichen und strukturellen Eigenschaften in vielen Bearbeitungsschritten technologische Herausforderungen, die für traditionelle Schriftkorpora als gelöst galten oder gar nicht erst bestanden. Im Folgenden stellen wir die sieben wichtigsten Arbeitsschritte bzw. Themen dar, für die es in der DEREKo-Verarbeitungspipeline so wie auch allgemein für IBK-Korpora derzeit noch keine befriedigende Lösung gibt.

Content Scraping: Wikipedia-Archive werden in sogenannten Dumps fortlaufend zur Verfügung gestellt, erreichen uns also in ähnlicher Form wie Zeitungsarchive. Für viele andere IBK-Genres muss aber ein jeweils spezifisches und Content Scraping implementiert werden, also ein Programm, das bestimmte Webseiten ggf. regelmäßig aufsucht, um bestimmte Inhalte - hier Textinhalte - aus ihnen zu extrahieren und in einer geeigneten Struktur abzulegen. Da die heute meist dynamisch generierten Webseiten keine stabile Präsenz aufweisen, müssen die Extraktionsroutinen praktisch permanent gewartet und überarbeitet werden.

Korpusmodellierung und -repräsentation: Das DEREKo-Textmodell basiert wie das Modell vieler anderer Referenzkorpora auf den Richtlinien der Text Encoding Initiative (TEI). Internetbasierte Kommunikation ist in den TEI-Guidelines jedoch bisher nicht modelliert. Das betrifft die Frage, was genau ein Text in IBK ist, bis hin zur Modellierung ihrer typischen dialogischen Organisation in Threads und Posts mit Antwort- und Adressierungsstrukturen. Verschiedene IBK-Korpusprojekte haben sich daher in der TEI Special Interest Group CMC zusammengeschlossen und ein Basismodell „<post> “ für die Repräsentation von Nutzerbeiträgen in IBK für ihre Korpora als sogenannte Customisierung umgesetzt (Abb. 4; vgl. Beißwenger et al. 2017b sowie Beißwenger in diesem Band). Die TEI CMC SIG arbeitet zurzeit daran, dass dieses Modell in die offizielle TEI aufgenommen wird (Beißwenger et al. i. Ersch.). 


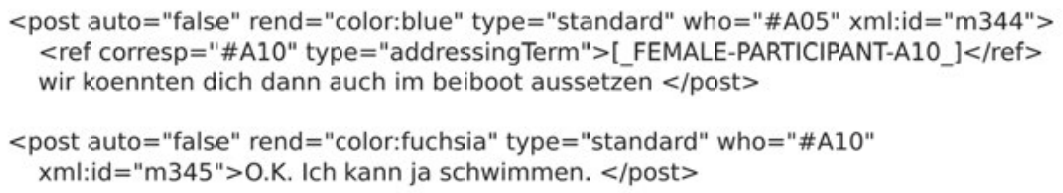

Abb. 4: Posts des Chatkorpus nach TEI SIG CMC

IBK-spezifische Metadaten wie Daten zur Plattform, zum Plattformbetreiber, zum Datum des Downloads bis hin zu einer Beschreibung der Kommunikationssituation aus Nutzersicht, also dessen, was ein Benutzer sieht und welche Funktionen ihm zur Verfügung stehen, sind in gängigen Metadatenschemata wie z.B. dem TEIHeader bisher nicht vorgesehen. Da die Features von Social Media permanent im Fluss sind, ist es umso wichtiger, derartige Informationen auch festzuhalten.

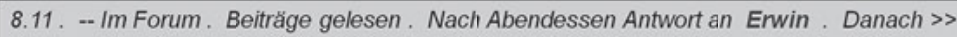

Abb. 5: Anonymisierung im Chatkorpus

Anonymisierung (Abb. 5): Oben haben wir beschrieben, dass in vielen IBK-Korpusprojekten wie auch für mündliche Korpora die Daten manuell anonymisiert werden. Dies ist aber für sehr große Textmengen, wie DEREKo sie anstrebt, nicht praktikabel. Wir benötigen eine automatische Anonymisierung, die den juristischen Anforderungen genügt und gleichzeitig den Nutzen der Korpora so wenig wie möglich einschränkt. NER-Algorithmen decken dabei nur einen Teil der Anforderungen $\mathrm{ab}$.

Normalisierung und Tokenisierung (Abb. 6): IBK-Texte zeichnen sich durch nicht-standardkonforme Sprache auf allen Ebenen der Grammatik inklusive der Orthografie aus. Um IBK-Korpora vollständig nutzbar zu machen, bedarf es einer Abbildung auf standard-orthografische Schreibungen (Normalisierung), da kein Nutzer beim Formulieren einer Suchanfrage alle vorkommenden Varianten voraus- 
ahnen kann. Auch einige Methoden für nachgelagerte Annotationen (z.B. Tagging, Anonymisierung, s.u.) basieren auf normalisierten Daten (vgl. Horbach et al. 2015).

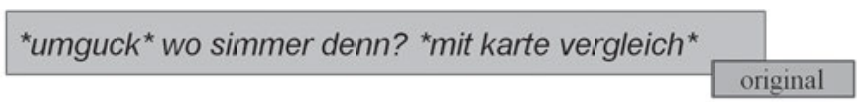

* umguck * wo sind wir denn? * mit Karte vergleich *

normalisierte Tokens

<w normal="sind wir" type="VVFIN" xml:id="m262.t5" $>$ simmer $\langle/$ w $>$

Abb. 6: Normalisierung im Chatkorpus

Tagging und linguistische Annotationen: Verfügbare Tagger und Parser für das Deutsche sind in der Regel auf Treebank-Annotationen von Zeitungstexten trainiert. Auf IBK-Daten haben sie eine entsprechend hohe Fehlerrate. Dies liegt nicht nur an dem Nicht-Standard-Charakter von IBK, sondern auch an den häufigen Vorkommen von IBK-spezifischen Einheiten wie Adressierungen, Emojis oder Hashtags. Für das POS-Tagging des Dortmunder Chatkorpus wurde ein auf Chat- und Forumsdaten nachtrainierter Tagger mit dem um CMC-Kategorien erweiterten Tagset STTS_IBK verwendet (Beißwenger et al. 2015b). Um dieses Forschungsfeld voranzubringen, werden weitere große Mengen von Trainingsdaten (manuell angefertigte und verifizierte syntaktische Annotationen) möglichst aller IBK-Genres benötigt (Beißwenger et al. 2016).

Abfrageschnittstellen: Die spezialisierten Abfrageschnittstellen der Referenzkorpora wie z.B. KorAP in Abbildung 4 sind derzeit aufgrund der typischen Lizenzbedingungen für Texte professioneller Schreiber so implementiert, dass sie für Suchanfragen als Treffer eine Menge von Textstellen maximal in Zitatgröße anzeigen. Für die Bestimmung des anzuzeigenden Ausschnitts werden Satzgrenzen und Paragraphengrenzen berücksichtigt; im Fall von IBK wäre aber die Post-Grenze eine viel sinnvollere Einheit. Andererseits ist die Zitatgröße für IBK oftmals eine unnötig starke Beschränkung, da vorliegende Lizenzen für IBK-Daten durchaus mehr Kontext anzuzeigen erlauben. Und schließlich gelten in den derzeitigen Recherchesystemen Einheiten wie eine Diskussionsseite oder ein Chat-Logfile als die Einheit „Text“, zu der man sich Metadaten anzeigen lassen kann, nach denen man auch den Bestand durchsuchen und sortieren kann. Auf die Metadaten eines Posts (wie Zeit, Autor, Adressierung) hingegen kann, obwohl in den Korpusdaten vorhanden, von der Benutzerin derzeit nicht zugegriffen werden. 
Für die Bearbeitung dieser Themen betreibt und sucht das Korpusausbauprojekt des IDS Kooperationen mit Forschungsprojekten in Europa oder auch mit Studierenden, die eine Abschlussarbeit in einem der Bereiche anfertigen möchten.

\section{IBK-Ressourcen am IDS}

\subsection{Dortmunder Chatkorpus}

Das Dortmunder Chatkorpus enthielt ursprünglich 480 Chat-Logfiles verschiedener Plattformen und Genres (Freizeitchats, Politikerchats, Seminarchats u.a.) aus den Jahren 2000 bis 2006 und wurde von Michael Beißwenger und Angelika Storrer, damals an der TU Dortmund, aufgebaut. In einem Kurationsprojekt 2015-2016 (Partner Universität Mannheim, TU Dortmund, BBAW, IDS) wurden die Daten für die CLARIN-D-Forschungsinfrastrukturen aufbereitet, im Einzelnen wurden die Daten in das Format CLARIN-D-TEI (für CMC) überführt und mit CMC-spezifischem POS-Tagging versehen (Beißwenger et al. 2015a). Außerdem wurde ein Rechtsgutachten über die Möglichkeit der Wiederveröffentlichung in den CLARIN-D-Infrastrukturen eingeholt, infolgedessen zehn Logfiles entfernt und die restlichen Daten anonymisiert wurden (Beißwenger et al. 2017a). Sie sind nun mit einem Umfang von 470 Chat-Logfiles in den Infrastrukturen von BBAW und IDS verfügbar.

Tab. 1: Dortmunder Chatkorpus 2.2

\begin{tabular}{ll}
\hline Inhalt & $\begin{array}{l}\text { Chatlogs aus den Jahren 2000-2006; Freizeitchats, Seminarchats, } \\
\text { Medienchats, u.v.m. }\end{array}$ \\
\hline Umfang & 470 Chatlogs \\
& 131.003 Posts \\
& 1.005 .166 Tokens \\
\hline Verfügbarkeit & Recherchierbar in COSMAS II \\
& CLARIN-D-TEI-Dateien herunterladbar aus dem IDS-Repositorium ${ }^{4}$ \\
\hline
\end{tabular}

4 https://repos.ids-mannheim.de/ 


\subsection{Usenet News-Korpus}

Das Usenet ist ein alter Teil des Internets, der auf dem sogenannten NNTP-Protokoll basiert und 1980 startete. Es ist organisiert in sogenannten Newsgroups, dabei handelt es sich um themenbezogene Diskussionsforen, in denen Threads zu Unterthemen gestartet werden können. Es gibt eine universelle Hierarchie von Newsgroups, so dass es theoretisch für jedes Thema genau eine Newsgroup gibt. ${ }^{5}$ Die Messages in einer Newsgroup werden auch News genannt. Das Usenet ist nicht proprietär, jeder kann mittels eines News-Clients (die meisten E-Mail-Programme können als News-Client konfiguriert werden) teilnehmen, es kann sogar jeder einen News-Server betreiben. Usenet News sind zweifellos nicht Social Media, gehören nicht einmal zum World Wide Web, sind aber definitiv IBK. Ihre Hochzeit war in den 1990er Jahren, das Usenet lebt aber weiterhin (vgl. Lüngen/Kupietz 2017 und Schlobinski in diesem Band).

Tab. 2: Usenet-News-Korpus

\begin{tabular}{ll}
\hline Inhalt & Alle Newsgruppen der de.-Hierarchie, 2013-2016 \\
\hline Umfang & 375 Newsgruppen \\
& 1 Million Messages \\
& 92 Millionen Tokens \\
\hline Verfügbarkeit & Recherchierbar in COSMAS II (bisher nur innerhalb des IDS) \\
\hline
\end{tabular}

\subsection{Meta.Tagesschau.de-Korpus}

In einer Kooperation mit der IDS-Abteilung Grammatik werden Nutzerkommentare zu Berichten auf tagesschau.de seit 2013 fortlaufend aus dem Web gesammelt. Zusätzlich wurde der Retrobestand 2009-2014 von dem Web-Archiv archive.org heruntergeladen. Die Daten wurden ursprünglich in dem BMBF-Projekt Analyse und Instrumentarium zur Beobachtung des Schreibgebrauchs im Deutschen (20132016) verwendet. Die Integration der Daten in DEREKo ist geplant, wenn eine rechtliche Klärung dafür grünes Licht gibt.

5 Beispiele von Namen deutschsprachiger Newsgroups: de.rec.musik.klassik, de.talk.jokes, de.rec.sport.motorsport.misc. 
Tab. 3: Korpus meta.tagesschau.de

\begin{tabular}{ll}
\hline Inhalt & Nutzerkommentare zu Berichten auf tagesschau.de ab 2009 \\
\hline Umfang & 75.000 Threads \\
& 2,5 Millionen Posts \\
& 175 Millionen Tokens \\
\hline Perspektive & Rechtliche Klärung, Integration in DEREKo \\
\hline
\end{tabular}

\subsection{Wikipedia-Korpora}

Die Online-Enzyklopädie Wikipedia (seit 2001) ist eine der am meisten besuchten Websites im WWW. Sie beinhaltet riesige Archive an kollaborativ erstelltem Textmaterial, vorwiegend in den beiden Genres Enzyklopädie-Artikel und OnlineDiskussionen (engl.talk pages). Wie wir gesehen haben, ist Wikipedia ein klassisches Soziales Medium, in dem die Merkmale Kommunikation und Kollaboration realisiert sind. Als IBK gelten jedoch nur die Diskussionen.

Seit 2005 wurden am IDS Wikipedia-Korpora in TEI konvertiert und in DEREKo integriert, seit 2011 werden alle zwei Jahre aus den aktuellen Wikipedia-Dumps neue Konvertierungen aller deutschsprachigen Artikel hergestellt und aufgenommen. Frühere Konvertierungen werden weiterhin vorgehalten, da nicht nur immer neue Texte hinzukommen, sondern vorhandene Artikel auch fortlaufend verändert werden.

So wie die deutschsprachige Wikipedia gewachsen ist, so sind mithin auch die abgeleiteten Korpora gewachsen; in der Konvertierung von 2017, welche die letzte Säule in Abbildung 7 anzeigt, sind 1,5 Milliarden Tokens erreicht, davon sind der orangefarbene Bereich die Artikel mit 870 Millionen Tokens bzw. 57\% der Konvertierung, die restlichen $43 \%$ entsprechend also Diskussionsseiten, die sich wiederum in Artikeldiskussionen und Nutzerdiskussionen aufteilen.

Tab. 4: Umfang der Wikipedia-Konvertierungen 2017

\begin{tabular}{llll}
\hline & Texte & Posts & Tokens \\
\hline Artikel & 2.065 .926 & $-/-$ & 873.182 .923 \\
\hline Artikeldisk. & 744.857 & 7.107 .696 & 349.075 .823 \\
\hline Nutzerdisk. & 603.374 & 5.895 .545 & 309.390 .966 \\
\hline Redundanzdisk. & 240 & 52.393 & 1.775 .975 \\
\hline$\Sigma$ & 3.414 .397 & 13.055 .634 & 1.533 .425 .687 \\
\hline
\end{tabular}




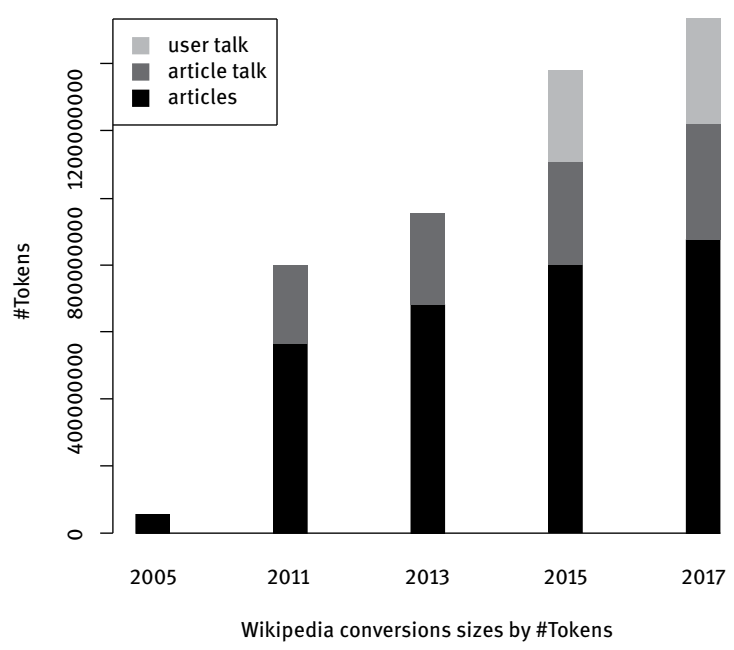

Abb. 7: Größen der Wikipedia-Konvertierungen

Alle deutschsprachigen Wikipedia-Korpora bis 2017 sind Teil von DEREKo und in COSMAS II recherchierbar. Außerdem sind alle deutschsprachigen WikipediaKorpora der Konvertierung 2017 in KorAP recherchierbar. Der IDS-Wikipedia-Konverter mit Dokumentation ist herunterladbar - damit können Nutzer eigene Wikipedia-Korpora aus Wikipedia-Dumps herstellen. Schließlich bieten wir auch analog hergestellte Wikipedia-Korpora für acht weitere europäische Sprachen an, die in IDS-Projekten eine Rolle spielen. Alle Wikipedia-Korpora sind auch als TEI-I5Dateien von unserem Download-Server herunterladbar.

\section{Eigenschaften von IBK-Korpora: Wikipedia-Diskussionen}

Die sprachlichen und funktionalen Unterschiede zwischen Wikipedia-Diskussionen und Wikipedia-Artikeln haben nicht zuletzt Herzberg/Storrer (2019) und Gredel (in diesem Band) herausgearbeitet - im Folgenden möchten wir auf Eigenschaften der Wikipedia-Diskussionen (Korpus WDD17) im Vergleich zum vollständigen Deutschen Referenzkorpus (DEREKo-Gesamt) eingehen. Die Leitfragen dabei sind: Was zeichnet die Wikipedia-Diskussionen im Vergleich zu DEREKo-Gesamt aus, was ist ihr Beitrag zu DEREKo-Gesamt und was sind ihre typischen Eigenschaften? Können Wikipedia-Diskussionen als typisch für Kommunikation in Social Media gelten? Und lohnt sich der Aufwand der Wikipedia-Korpusaufbereitung? 
$<$ Zah $>$ CEST du ich CET Artikel nicht Du ist Ich Wikipedia hier dir Hallo hast mal Mär dich Apr kannst bitte mir Feb auch Aug ja Jul Okt Sep Dez Jun Nov Jan Grüße Gruß dass habe da aber es oder Bitte deine - Wenn Benutzernamen was man wenn Benutzer Benutzerkonten mich kann Beitrag Danke Quelle Diskussian das nur Dein dann Dir sollte Mai Änderungen zB diese Quellen Artikels wäre gelöscht keine signierter Text Abschnitt deinen Deine deiner Link also Wikimedia wirst Seite findest

Abb. 8: Typischste Wörter in den Wikipedia-Diskussionen im Vergleich zu DEREKo-Gesamt (sortiert nach ihrem Beitrag zur Kullback-Leibler-Divergenz)

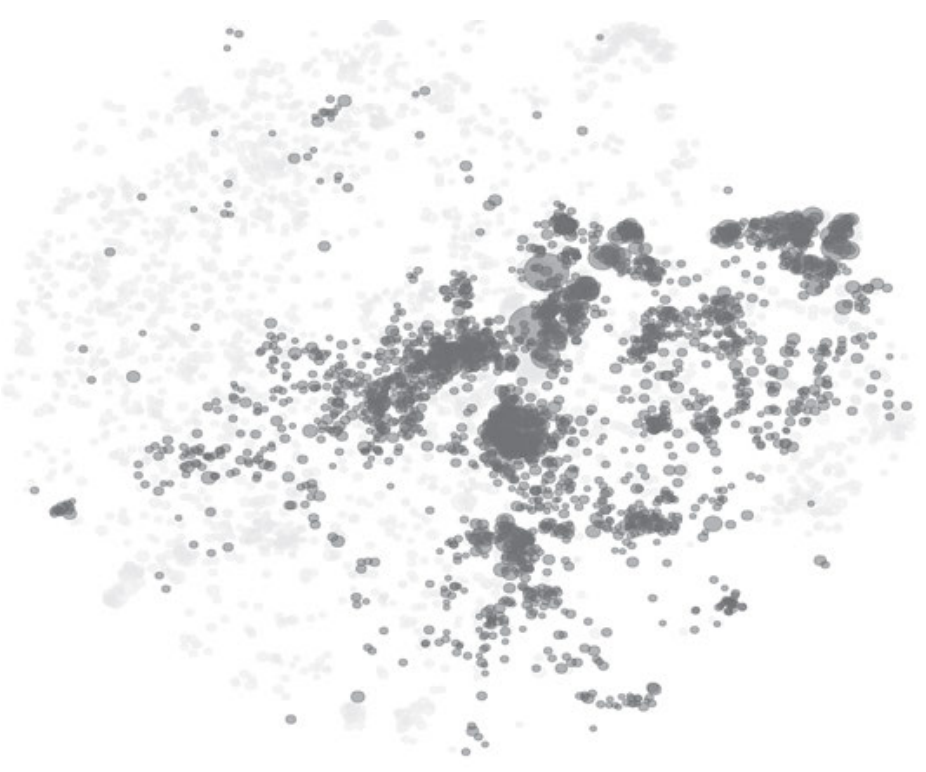

Abb. 9: Projektion des Word-Embedding Modells trainiert auf WDD17 und DEREKo-Gesamt

Unter den im Kontrast zu DeReKo-Gesamt typischsten Wörtern findet man zum einen, wie man es für IBK erwartet, Pronomina und Verbformen und der 1. und 2. Person Singular und Grußformeln, zum anderen spezifisches Vokabular des Editierens von Online-Enzyklopädieartikeln wie Benutzerkonten, Beitrag, Artikel, Änderung (Abb. 8). Interessanter wird es, wenn man sich mit dem Tool DeREKoBubbles (Fankhauser/Kupietz 2019) die jeweils typischsten Wörter im distributio- 
nell semantischen Kontext ansieht. Dieses Tool arbeitet mit einem zunächst auf DEREKo-Gesamt und dann auf WDD17 trainierten Word-Embedding-Modell. Die eigentlich 200-dimensionalen Wortvektoren wurden hier mit Hilfe von T-SNE so weit wie möglich Topologie-erhaltend auf eine Karte projiziert, so dass Nähe auf der Karte distributionelle Ähnlichkeit bedeutet (Abb. 9). Die dunklen Blasen stehen dabei für Vektoren aus WDD17. Man sieht, dass sie sich in bestimmten Bereichen klumpen, dabei aber nur bestimmte Teile des gesamten Raums, den DEREKo aufspannt (helle Blasen), abdecken. Das liegt daran, dass in Wikipedia einige pressespezifische Themen (wie Lokalpolitik, Veranstaltungen, Klatsch) keine Rolle spielen, aber auch daran, dass die für Diskussionen typischen Wörter sich hier textsortenspezifisch auf bestimmte Bereiche konzentrieren.

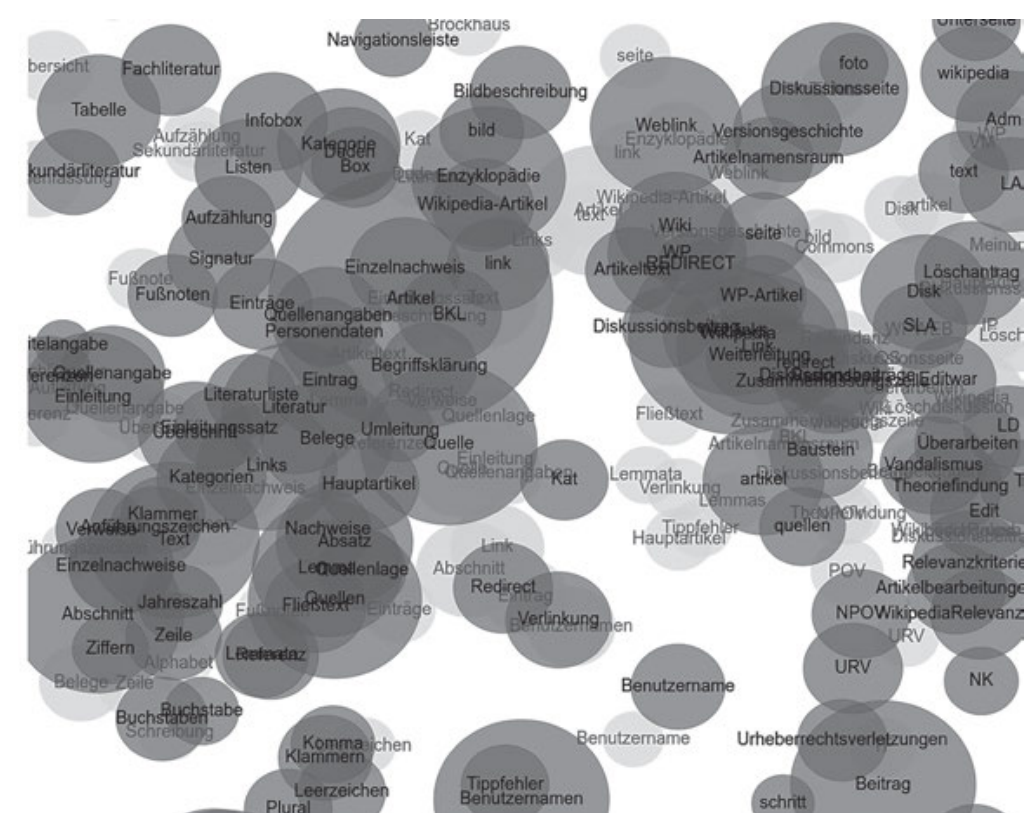

Abb. 10: Zoom auf Cluster

In dem Beispielcluster in Abbildung 10 sehen wir Vokabular, das sich auf Wikipediastrukturen und die Editionssituation bezieht wie Fachliteratur, Kategorie, bearbeiten; auch das von Gredel (in diesem Band) identifizierte Werkstattvokabular der Wikipedia findet sich in diesem Cluster (z.B. Theoriefindung, POV, Relevanz). Ein weiteres Cluster (ohne Abbildung) liefert ein reiches Inventar von Adverbien, die in der Argumentation der Textkritik gebräuchlich sind (allenfalls, offenkundig, annähernd, wohlgemerkt, ...) 
Um weitere offensichtliche Cluster dieser Art zu finden, hat sich die Differenz der paradigmatischen Projektion als nützlich erwiesen. Dabei handelt es sich um die Differenz zwischen der Anzahl der Nachbarn aus DEREKo-Gesamt eines Vektors und der Anzahl der Nachbarn aus WDD17 desselben Vektors in einem bestimmten Umkreis im distributionell semantischen Raum. Die paradigmatische Projektion (nach Entropie) ist in Tabelle 5 in der fünften Spalte wiedergegeben, nach der diese Tabelle auch sortiert ist. Dass hier die Form unglücklich besonders hoch bewertet ist, bedeutet also, dass die Anzahl ihrer Nachbarn in DEREKo im Vergleich zur Anzahl ihrer Nachbarn in WDD17 in demselben Raum besonders unterschiedlich ist, d.h. dass diese Form sich in ihrer Bedeutung in DEREKo-Gesamt besonders stark von der Bedeutung, die sie in den WikipediaDiskussionen hat, unterscheidet. Die Sortierung nach der paradigmatischen Projektion legt also die Hypothese nahe, das Wörter mit ansonsten vielfältigen Verwendungskontexten in DEREKo in den Wikipedia-Diskussionen hauptsächlich im Kontext der Textkritik verwendet werden, vgl. neben unglücklich auch die Form Schrott.

Tab. 5: Wortvektoren aus WDD17 nach paradigmatischer Projektion

\begin{tabular}{lrrrrrr}
\hline word & fpm & \multicolumn{1}{c}{ kld } & \multicolumn{1}{c}{ ent } & \multicolumn{1}{c}{ entd } & \multicolumn{1}{c}{ dist } & \multicolumn{1}{c}{ dist2d } \\
\hline unglücklich & 21.21 & 22.77 & 3.306 & 3.306 & 0.2163 & 0.291 \\
\hline Schrott & 5.84 & 2.76 & 2.502 & 2.502 & 0.2214 & 0.120 \\
\hline Singles & 4.85 & -1.71 & 2.238 & 2.238 & -0.1769 & -0.095 \\
\hline Umstellungen & 1.35 & -0.61 & 2.036 & 2.036 & -0.2676 & -0.123 \\
\hline LG & 71.63 & 188.90 & 2.027 & 2.027 & 0.3611 & 1.479 \\
\hline unhaltbar & 4.03 & 1.05 & 2.019 & 2.019 & 0.2941 & 0.755 \\
\hline Prima & 8.71 & 18.21 & 1.952 & 1.952 & 0.2535 & 0.325 \\
\hline Regiment & 2.67 & -0.82 & 1.925 & 1.925 & -0.1410 & -0.065 \\
\hline Jo & 11.26 & 14.50 & 1.921 & 1.921 & 0.1998 & 20.210 \\
\hline Setzen & 3.41 & 2.69 & 1.921 & 1.921 & 0.2072 & 0.148 \\
\hline vorherige & 13.24 & 21.64 & 1.777 & 1.777 & 0.1172 & 0.130 \\
\hline morgen & 47.71 & -54.42 & 1.897 & 1.764 & -0.2682 & -0.104 \\
\hline genauer & 77.97 & 153.60 & 1.752 & 1.752 & 0.0734 & 0.011 \\
\hline Trennung & 28.92 & 12.41 & 1.714 & 1.714 & 0.1055 & 0.200 \\
\hline
\end{tabular}




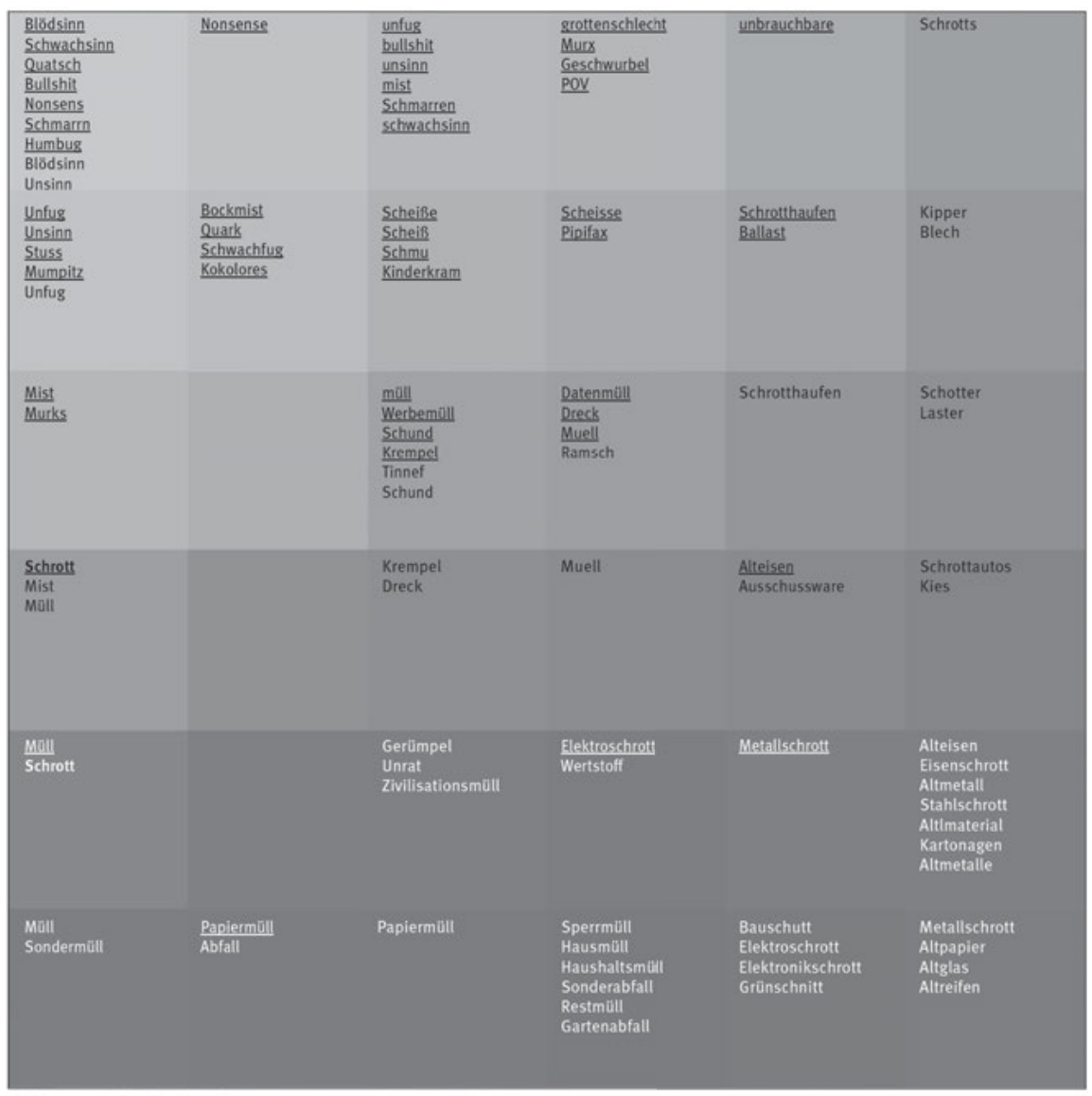

WDD17 DEREKo-Gesamt

Abb. 11: Selbstorganisierende Karte in der DeReKoVeCs: Vergleich der Ähnlichkeiten von Schrott in WDD17 und DEREKo-Gesamt

Mit diesem Befund können wir uns Schrott im Mikrovergleich unter Verwendung von DeReKoVecs (Kupietz et al. 2018) anschauen: In Abbildung 11 sind Schrott und seine 50 nächsten Nachbarn jeweils in WDD17 und in DEREKo-Gesamt auf einer selbstorganisierenden Karte zu sehen; dabei haben sich oben links in dem helleren Quadranten diejenigen Formen angeordnet, die in WDD17 in besonders ähnlicher Weise zu Schrott verwendet werden wie Blödsinn, Schwachsinn, Quatsch (unterstrichen), während sich in dem von DeReKo-Gesamt dominierten Quadranten unten rechts Formen wie Metallschrott, Altpapier und Altglas als semantisch nächste Nachbarn finden (nicht unterstrichen). Die Hypothese, dass bestimmte 
Wörter in den Wikipedia-Diskussionen Lesarten aus dem Bereich der Textkritik annehmen, bestätigt sich also.

Entsprechende Ergebnisse zeigt schließlich auch eine Kookkurrenzanalyse der nächsten syntagmatischen Nachbarn von Schrott in WDD17 (in Abb. 12, sortiert nach der Log-Dice-Score-Differenz zwischen WDD17 und DEREKo-Gesamt), die textkritische Kollokationen wie unbelegter, völliger oder reinster Schrott liefert, oder auch Schrott fabrizieren, Schrott verzapfen. ${ }^{6}$

Als Zwischenfazit dieses korpuslinguistischen Vergleichs der Wikipedia-Diskussionen gegenüber DEREKo-Gesamt in Mikro- und Makroanalysen lässt sich festhalten: Wikipedia-Diskussionen weisen sprachliche Aspekte von Social MediaKommunikation auf, sind dabei aber aufgabenspezifisch mit einem Fokus auf Textkritik versehen. Da sie außerdem nur einen begrenzten Autorenkreis haben, können sie als Ganzes eher nicht als typisches Beispiel für Social Media-Kommunikation dienen, wenn man davon ausgeht, dass typische Social Media-Kommunikation (wie Interaktionen auf Facebook, Twitter oder WhatsApp) thematisch und funktional offen ist. Andererseits gilt weiterhin, dass Wikipedia-Diskussionen eine große und weiter wachsende Ressource unter einer liberalen Lizenz darstellen, dass sie reich an Metadaten sind und dass sie in vielen Sprachen verfügbar sind. Viele einzelne linguistisch relevante Aspekte können durchaus anhand von Wikipedia-Diskussions-Korpora qualitativ und quantitativ untersucht werden, wie politische Diskurse zu verschiedenen Themen (vgl. Gredel 2018), kontrastiv-grammatische Aspekte (Augustin 2016), Höflichkeit oder Konflikte (Ho-Dac et al. 2017).

\section{Perspektiven}

IBK-Korpora mit ihrem user-generated content bieten viele neue Möglichkeiten für die Untersuchung der Gegenwartssprache, beispielsweise für die Detektion und Analyse von Neologismen, für die Rechtschreibbeobachtung oder für die Diskursanalyse. Das Deutsche Referenzkorpus strebt daher an, den Bestand an IBK weiter auszubauen. In diesem Beitrag haben wir herausgearbeitet, dass nicht wie in allgemeinen Web-Korpora ein breit gestreutes Sampling von IBK auf Vorrat durchgeführt werden kann: Es gibt rechtliche Problematiken für viele Subgenres, es gibt jeweils eine große Anzahl potenzieller Lizenzpartner, und sie erfordern eine jeweils spezifische und aufwändige Aufbereitung. DEREKo plant daher, in spezifischen

6 Einige der Kollokate in Abbildung 12 erscheinen offenbar aufgrund von Vorkommen von Schrott als Tippfehler des Wortes Schrot. 
Projekten und in Kooperationen $\mathrm{zu}$ bestimmten Subgenres weitere IBK-Korpora aufzubauen. Hierzu besteht derzeit eine Kooperation mit dem Projekt MoCoDa2 zu Privatchats. Für darüber hinausgehende Erweiterungen sind ebenfalls Kooperationen erforderlich, beispielsweise dem Zentrum für digitale Lexikographie der deutschen Sprache (ZDL) zur Akquisition von Blogdaten, mit dem Leibniz-Institut für Sozialwissenschaften (GESIS) zu Twitter-Daten und mit dem Forschungszentrum für Grundlagen- und anwendungsorientierte Forschung (L3S) zum Aufbau von vorstrukturierten (z.B. nach IBK, nach Lizenz) Webkorpora. Schließlich werden wir die am IDS betriebenen Korpusströme (Wikipedia, Usenet, TagesschauKommentare) weiterhin integrieren.

\section{Literatur}

Augustin, Hagen (2016): Quantitative Untersuchungen zum deutschen Vorfeld und seinen Äquivalenten in sechs verschiedensprachigen Wikipedia-Korpora. In: Dalmas, Martine/ Fabricius-Hansen, Cathrine/Schwinn, Horst (Hg.): Variation im europäischen Kontrast. Untersuchungen zum Satzanfang im Deutschen, Französischen, Norwegischen, Polnischen und Ungarischen. (= Konvergenz und Divergenz 5). Berlin/Boston, S. 9-52.

Barbaresi, Adrien/Würzner, Kay-Michael (2014): For a fistful of blogs: Discovery and comparative benchmarking of republishable German content. In: Workshop Proceedings of the 12th KONVENS 2014, 8.-10. Oktober 2014. Hildesheim, S. 2-10.

Beißwenger, Michael/Bartsch, Sabine/Evert, Stefan/Würzner, Kay-Michael (2016): EmpiriST 2015: A shared task on the automatic linguistic annotation of computer-mediated communication and web corpora. In: The 54th Annual Meeting of the Association for Computational Linguistics. Proceedings of the 10th Web as Corpus Workshop (WAC-X) and the EmpiriST Shared Task. Stroudsburg: As-sociation for Computational Linguistics (ACL Anthology W16-2606), 12. August 2016. Berlin, S. 44-56.

Beißwenger, Michael/Fladrich, Marcel/Imo, Wolfgang/Ziegler, Evelyn (2018): News from the MoCoDa2 corpus: A design and web-based editing environment for collecting and refining data from private CMC interactions. In: Vandekerckhove, Reinhild/Fišer, Darja/Hilte, Lisa (Hg.): Proceedings of the 6th Conference on Computer-Mediated Communication (CMC) and Social Media Corpora (CMC-corpora 18), 17.-18. September 2018, University of Antwerp. Antwerpen, S. 10-14.

Beißwenger, Michael/Bartz, Thomas/Storrer, Angelika/Westpfahl, Swantje (2015b): Tagset und Richtlinie für das PoS-Tagging von Sprachdaten aus Genres internetbasierter Kommunikation. In: EmiriST 2015: Shared Task des Empirikom-Netzwerks zur automatischen linguistischen Annotation deutschsprachiger internetbasierter Kommunikation. Internet: https://docs.google.com/viewer?a=v\&pid=sites\&srcid=ZGVmYXVsdGRvbWFpbn xlbXBpcmlzdDIwMTV8Z3g6OWQ0YWNmZWE5OTUzMjE (Stand: 22.8.2019).

Beißwenger, Michael/Herzberg, Laura/Lüngen, Harald/Wigham, Ciara R. (i. Ersch.): CMC-core: A basic schema for encoding CMC corpora in TEl. Poster abstract for the 7th Conference on Computer-Mediated Communication and Social Media Corpora. Cergy-Pontoise. 
Beißwenger, Michael/Ehrhardt, Eric/Horbach, Andrea/Lüngen, Harald/Steffen, Diana/Storrer, Angelika (2015a): Adding value to CMC corpora: CLARINification and part-of-speech annotation of the Dortmund Chat Corpus. In: Beißwenger, Michael/Zesch, Torsten (Hg.): NLP4CMC 2015. Proceedings of the 2nd Workshop on Natural Language Processing for Computer-Mediated Communication/Social Media, 29. September 2015, University of Duisburg-Essen. Essen, S. 12-16.

Beißwenger, Michael/Lüngen, Harald/Schallaböck, Jan/Weitzmann, John H./Herold, Axel/ Kamocki, Pawel/Storrer, Angelika/Wildgans, Julia (2017a): Rechtliche Bedingungen für die Bereitstellung eines Chat-Korpus in CLARIN-D: Ergebnisse eines Rechtsgutachtens. In: Beißwenger, Michael (Hg.): Empirische Erforschung internetbasierter Kommunikation. (= Empirische Linguistik/Empirical Linguistics 9). Berlin/New York, S. 7-46.

Beißwenger, Michael/Wigham, Ciara R./Etienne, Carole/Grumt Suárez, Holger/Herzberg, Laura/Fišer, Darja/Hinrichs, Erhard/Horsmann, Tobias/Karlova-Bourbonus, Natali/ Lemnitzer, Lothar/Longhi, Julien/Lüngen, Harald/Ho-Dac, Lydia-Mai/Parisse, Christophe/ Poudat, Céline/Schmidt, Thomas/Stemle, Egon W./Storrer, Angelika/Zesch, Torsten (2017b): Connecting resources: Which issues have to be solved to integrate CMC corpora from heterogeneous sources and for different languages? In: Stemle, Egon W./Wigham, Ciara R. (Hg.): Proceedings of the 5th Conference on CMC and Social Media Corpora for the Humanities (CMC-corpora 17), 3.-4. Oktober 2017, Eurac Research Italy. Bozen, S. 52-55.

Bodmer Mory, Franck (2014): Mit COSMAS II ,in den Weiten der IDS-Korpora unterwegs“. In Steinle, Melanie/Berens, Franz-Josef (Red.): Ansichten und Einsichten. 50 Jahre Institut für Deutsche Sprache. Mannheim, S. 376-385.

Cann, Alan/Dimitriou, Konstantia/Hooley, Tristram (2011): Social media: A guide for researchers. London: Research Information Network. Internet: http://hdl.handle. net/10545/196715 (Stand: 22.8.2019).

DFG-Handreichung (2013a): Informationen zu rechtlichen Aspekten bei der Handhabung von Sprachkorpora. In: Deutsche Forschungsgemeinschaft e.V. Internet: https://www.dfg.de/ download/pdf/foerderung/grundlagen_dfg_foerderung/informationen_fachwissenschaften/geisteswissenschaften/standards_recht.pdf (Stand: 22.8.2019).

DFG-Handreichung (2013b): Empfehlungen zu datentechnischen Standards und Tools bei der Erhebung von Sprachkorpora. In: Deutsche Forschungsgemeinschaft e.V. Internet: https:// www.dfg.de/download/pdf/foerderung/grundlagen_dfg_foerderung/informatio-nen_ fachwissenschaften/geisteswissenschaften/standards_sprachkorpora.pdf (Stand: 22.8.2019)

Diewald, Nils/Hanl, Michael/Margaretha, Eliza/Bingel, Joachim/Kupietz, Marc/Bański, Piotr/ Witt, Andreas (2016): KorAP Architecture - Diving in the deep sea of corpus data. In: Calzolari, Nicoletta/Choukri, Khalid/Declerck, Thierry/Goggi, Sara/Grobelnik, Marko/ Maegaard, Bente/Mariani, Joseph/Mazo, Helene/Moreno, Asuncion/Odijk, Jan/Piperidis, Stelios (Hg.): Proceedings of the 10th International Conference on Language Resources and Evaluation (LREC 2016), 23.-28. Mai 2016, Portorož, Slovenia. Paris, S. 3586-3591.

Fankhauser, Peter/Kupietz, Marc (2019): Analyzing domain specific word embeddings for a large corpus of contemporary German. In: Proceedings of the 10th International Corpus Linguistics Conference (CL2019), 22.-26. Juli 2019, Wales, Cardiff University. Cardiff.

Fišer, Darja/Beißwenger, Michael (Hg) (2017): Investigating computer-mediated communication: Corpus-based approaches to language in the digital world. Ljubljana.

Fišer, Darja/Ljubešić, Nikola/Erjavec, Tomaž (2018): The Janes project: Language resources and tools for Slovene user generated content. In: Language Resources and Evaluation, S. 1-24. Internet: https://link.springer.com/article/10.1007/s10579-018-9425-z (Stand: 28.8.2019). 
Frey, Jennifer-Carmen/Glaznieks, Aivars/Stemle, Egon W. (2016): The DiDi Corpus of South Tyrolean CMC Data: A multilingual corpus of Facebook texts. In: Corazza, Anna/ Montemagni, Simone/Semeraro, Giovanni (Hg.): Proceedings of the 3rd Italian Conference on Computational Linguistics CLiC-it 2016. 5.-6. Dezember 2016. Neapel/Turin, S. 157-161.

Gredel, Eva (2018): Digitale Diskurse und Wikipedia. Wie das Social Web Interaktion im digitalen Zeitalter verwandelt. (= DIALOGE 1). Tübingen.

Grumt Suárez, Holger/Karlova-Bourbonus, Natali/Lobin, Henning (2016): Compilation and annotation of the discourse-structured Blog Corpus for German. In: Beißwenger, Michael/ Fišer, Darja (Hg): Proceedings of the 4th Conference on CMC and Social Media Corpora for the Humanities, 27.-28. September 2016, Faculty of Arts of the University of Ljubljana. Ljubljana, S. 26-29.

Herring, Susan C. (2004): Slouching toward the ordinary: Current trends in computer-mediated communication. In: New Media \& Society 6, 1, S. 26-36.

Herring, Susan C. (2007): A faceted classification scheme for computer-mediated discourse. In: Language@Internet 4, S. 1-37.

Herzberg, Laura/Storrer, Angelika (2019): Investigating OKAY across genres, modes and languages: A corpus-based study on German and French. In: Cahiers du Laboratoire de Recherche sur le Langage 8, S. 149-176.

Ho-Dac, Lydia-Mai/Laippala, Veronika (2017): Le corpus WikiDisc: Ressource pour la caractérisation des discussions en ligne. In: Wigham, Ciara R./Ledegen, Gudrun (Hg.): Corpus de communication médiée par les réseaux: Construction, structuration, analyse. Paris, S. 107-124.

Ho-Dac, Lydia-Mai/Laippala, Veronika/Poudat, Céline/Tanguy, Ludovic (2017): Exploring Wikipedia talk pages for conflict detection. In: Fišer/Beißwenger (Hg), S. 146-171.

Horbach, Andrea/Thater, Stefan/Steffen, Diana/Fischer, Peter M./Witt, Andreas/Pinkal, Manfred (2015): Internet corpora: A challenge for linguistic processing. In: DatenbankSpektrum 15, 1, S. 41-47.

Kamocki, Pawet/Kinder-Kurlanda, Katharina/Kupietz, Marc (2016): „One does not simply share data": Organisational and technical remedies to legal constraints in research data sharing - building bridges between Digital Humanities and the Social Sciences. Abstract for the Panel Witt, Andreas/Kamocki, Pawet: When DH meets law: Problems, solutions, perspectives. In: Digital Humanities 2016, Conference Abstracts, Jagiellonian University \& Pedagogical University Kraków, 11.-16. Juli 2016. Krakau, S. 104-108.

Kupietz, Marc/Lüngen, Harald/Kamocki, Pawet/Witt, Andreas (2018): The German Reference Corpus DeReKo: New developments - new opportunities. In: Calzolari, Nicoletta/Choukri, Khalid/Cieri, Christopher/Declerck, Thierry/Goggi, Sara/Hasida, Koiti/Isahara, Hitoshi/ Maegaard, Bente/Mariani, Joseph/Mazo, Hélène/Moreno, Asuncion/Odijk, Jan/Piperidis, Stelios/Tokunaga, Takenobu (Hg.): Proceedings of the 11th International Conference on Language Resources and Evaluation (LREC 2018), 7.-12. Mai 2018, Miyazaki, Japan. Paris, S. 4353-4360.

Ljubešić, Nikola/Erjavec, Tomaž/Fišer, Darja (2017): Wikipedia talk corpus Janes-Wiki 1.0. In: Slovenian language resource repository. Korpusressource. Internet: http://hdl.handle. net/11356/1137 (Stand: 22.8.2019).

Lüngen, Harald/Kupietz, Marc (2017): CMC Corpora in DeREKo. In: Bański, Piotr/Kupietz, Marc/ Lüngen, Harald/Rayson, Paul/Biber, Hanno/Breiteneder, Evelyn/Clematide, Simon/ Mariani, John/Stevenson, Mark/Sick, Theresa (Hg.): Proceedings of the Workshop on 
Challenges in the Management of Large Corpora and Big Data and Natural Language Processing (CMLC-5+BigNLP 2017) including the papers from the Web-as-Corpus (WAC-XI) guest section. Birmingham, 24. Juli 2017. Mannheim, S. 20-24.

Margaretha, Eliza/Lüngen, Harald (2014): Building linguistic corpora from Wikipedia articles and discussions. In: Journal for Language Technologie and Computational Linguistics (JLCL) 29, 2: Special issue on building and annotating corpora of computer-mediated communication. Issues and challenges at the interface between computational and corpus linguistics, S. 59-82.

Poudat, Céline/Grabar, Natalia/Kun, Jin/Paloque-Berges, Camille (2015): Corpus wikiconflits, conflits dans le Wikipédia francophone. In: Chanier, Thierry (Hg.): Banque de corpus CoMeRe. Nancy. Internet: https://hdl.handle.net/11403/comere/cmr-wikiconflits (Stand: 22.8.2019).

Scheffler, Tatjana (2017): Conversations on Twitter. In: Fišer/Beißwenger (Hg), S. 124-145.

Siebenhaar, Beat (2018): Funktionen von Emojis und Altersabhängigkeit ihres Gebrauchs in der WhatsApp-Kommunikation. In: Ziegler, Arne (Hg.): Jugendsprachen: Aktuelle Perspektiven internationaler Forschung/Youth languages: Current perspectives of international research. 2 Bde. Berlin, S. 749-772.

Storrer, Angelika (2018): Interaktionsorientiertes Schreiben im Internet. In: Deppermann, Arnulf/Reineke, Silke (Hg.): Sprache im kommunikativen, interaktiven und kulturellen Kontext. (= Germanistische Sprachwissenschaft um 2020 3). Berlin/Boston, S. 219-244.

Ueberwasser, Simone/Stark, Elisabeth (2017): What's up, Switzerland? A corpus-based research project in a multilingual country. In: Linguistik Online 84, 5, S. 105-126. 